\title{
La cuadratura de la parábola según Thabit: una forma de análisis a partir de resultados cinemáticos
}

Thabit's parábola quadrature: an approach through kinematical results

\author{
C. H. Wörner*1
}

${ }^{1}$ Programa de Magister en Didáctica de las Ciencias Experimentales, Pontificia Universidad Católica de Valparaíso, Av. Brasil 2950, Valparaíso 2340000, Chile

Recibido en 02 de Noviembre, 2017. Aceptado en 06 de Diciembre, 2017

\begin{abstract}
Se revisa el método que usó Thabit para encontrar el área de un segmento de parábola. Para ello, se utilizan los resultados obtenidos rutinariamente en la enseñanza del lanzamiento de proyectiles en un campo uniforme. Este tratamiento enfatiza la relación entre física y matemáticas y también recrea algunos argumentos históricos anteriores a la aparición del cálculo infinitesimal.
\end{abstract}

Palabras clave: Cuadratura de la parábola, cinemática, ciencia árabe.

The Thabit's area formula of a segment of a parabola is reconsidered. Use is made of the properties of the projectile launch describing a parabolic trajectory, as routinely exposed in introductory Physics courses. This treatment emphasizes the relationship between Mathematics and Physics, and it is also a recreation for some historic pre-calculus arguments

Keywords: Parabola's quadrature, kinematics, Arabic science.

\section{Introducción}

Este trabajo señala la posibilidad didáctica de conectar geometría y física. Esta relación se origina en la tradición griega y aparece escrita desde los trabajos de Galileo.

El asunto en comento es la demostración de la cuadratura de la parábola por Thabit. En tiempos del esplendor de la cultura árabe medioeval, se posiciona la figura de Al-Sabi Thabit ibn Qurra al-Harrani (826- 901 AC), quién estudió un sinnúmero de cuestiones relacionadas con lo que hoy llamaríamos la geometría griega.

Thabit fue un prominente científico árabe, cuyas contribuciones a las matemáticas y la astronomía fueron notables en tiempos en que la ciencia brillaba en los países árabes en la época medioeval (para nuestra cronología histórica occidental). Algunos ejemplos de sus contribuciones son la extensión del teorema de Pitágoras [1] y sus trabajos sobre números amigables [2]. El legado estos estudiosos "prerrenacentistas" es a menudo olvidado en tiempos contemporáneos.

Digamos que la cuestión de la cuadratura de la parábola fue un problema inicialmente resuelto por Arquímedes, quién formuló dos demostraciones radicalmente diferentes - una física y otra matemática- durante el siglo II dC [3]. Es decir, ha transcurrido un milenio hasta la aparición de la demostración de Thabit [4]. Aparentemente Thabit conocía algunos de los resultados geométricos de Arquímedes pero ignoraba su tratamiento de la cuadratura

*Endereço de correspondência: cworner@ucv.cl de la parábola. El problema resurge -ahora en Occidenteen el renacimiento. Galileo mismo [5] lo menciona en la discusión de la influencia de la sección sobre la tenacidad de una viga sólida, en la primera parte de su seminal trabajo "Dos Nuevas Ciencias". Su discípulo Torricelli [6] trata profusamente el tema y brillantes matemáticos como Wallis [7] y Fermat y Pascal [8] lo analizaron con diferentes argumentos anteriores al desarrollo del cálculo infinitesimal. Es interesante recorrer la línea del tiempo que se muestra en la Fig 1.

Es posible especular que la búsqueda de la fórmula para la cuadratura de la parábola, está en la búsqueda de la fórmula para la cuadratura del círculo, una de la cuestiones clásicas no resueltas por la geometría griega. Una pregunta que cubre ¡milenios de investigación matemática!

El método que usaremos en esta nota hace uso de las ecuaciones básicas de la caída libre, específicamente los resultados que se obtienen habitualmente en el estudio del lanzamiento horizontal de proyectiles en un

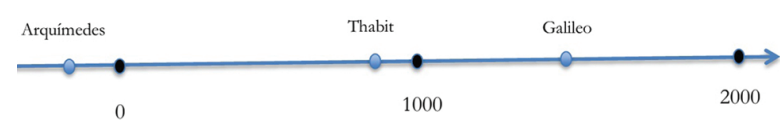

Figura 1: Línea del tiempo. 
campo gravitacional (constante), como el que existe en la cercanía terrestre.

Razonamientos simples describen la trayectoria parabólica como la "composición" de dos movimientos independientes, uno horizontal, con velocidad uniforme $v$ y otro, una caída libre con aceleración $g$

La Fig. 2 muestra estos dos movimientos básicos, es decir, el movimiento horizontal uniforme y el acelerado. El eje temporal $(X)$ está dividido en intervalos iguales, mostrando dos hechos que usaremos en nuestro desarrollo posterior. i) a tiempos iguales, la distancia total recorrida es proporcional a los tiempos al cuadrado y ii) las distancias parciales son proporcionales a la progresión de números impares. Galileo fue quien primero enunció estas propiedades en su obra seminal "Las Dos Nuevas Ciencias" [5]. Esta dos leyes pueden verificarse con experimentos simples (por ejemplo, ver Ref. 9).

\section{La demostración de Thabit}

La originalidad de la demostración de Thabit está en la utilización de las propiedades de la parábola recién mencionadas en el párrafo anterior. El utiliza una división del área de la parábola en trapecios con diferentes pero no arbitrarias- alturas. De hecho, dicha división trapezoidal sique la secuencia de los números impares (ver Fig. 3)

Para simplificar el desarrollo, consideremos una parábola descrita por $y=x^{2}$. Esta ecuación corresponde trivialmente a la forma paramétrica , $x=t ; y=t^{2}$ que es la forma adimensional de las ecuaciones cinemáticas $x=v t$; $y=(1 / 2) g t^{2}$ (nótese que el eje $Y$ apunta hacia abajo en la Fig. 2 y hacia arriba en la Fig. 3). Llamemos $h$ a la altura de todo el segmento de parábola, es decir $0 \leq y \leq h$. Además, $-\sqrt{h} \leq x \leq+\sqrt{h}$

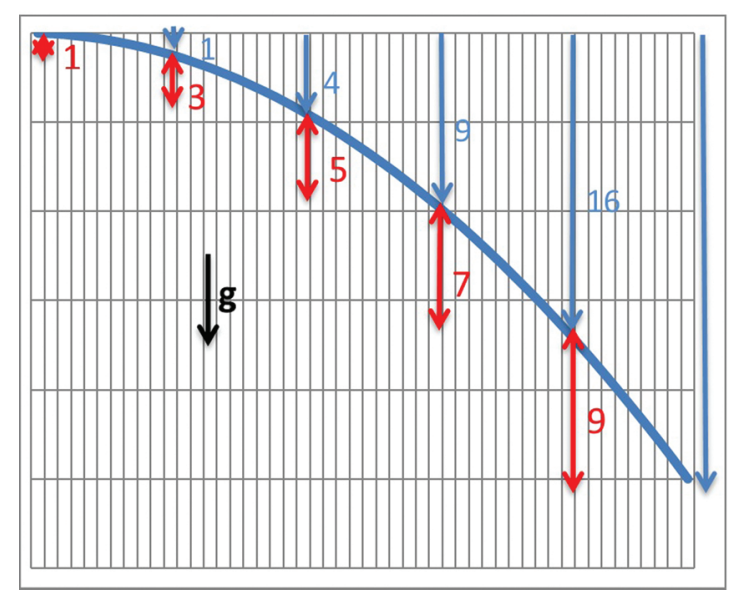

Figura 2: Caída libre parabólica con velocidad inicial horizontal. Los números indican las distancias totales recorridas (líneas azules) y la parciales (rojas).

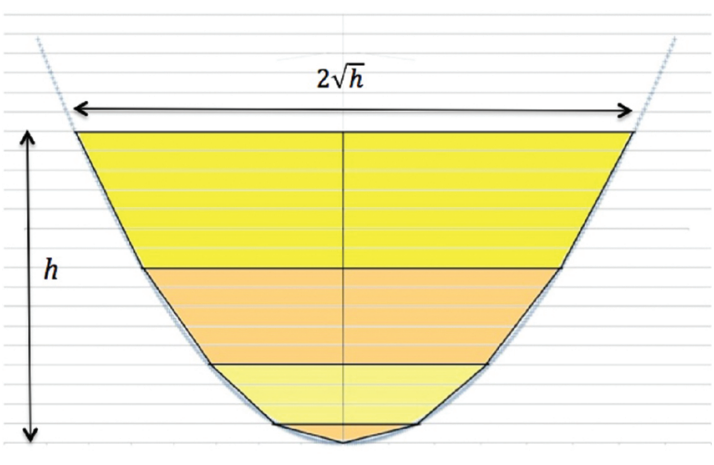

Figura 3: La partición de Thabit para $N=4$. En este caso $h=16$.

El argumento original usaba el "método de extenuación' 1 (que consistía en inscribir y circunscribir dos conjuntos de trapecios cuyas áreas se aproximaban por defecto o exceso al área total a calcular). En cambio aquí usaremos el método algebraico más simple y directo.

El problema -que se reduce a un asunto aritméticopuede ser tratado por métodos rigurosos, pero en términos didácticos parece mejor un proceso semi-heurístico.

Tomaremos el caso particular expuesto en le Fig. 3 que corresponde a una partición con $N=4$ con la división en 1, 3, 5 y 7 segmentos trapezoidales. Aplicando la regla para calcular las áreas del trapecio, podemos escribir la suma de las áreas trapezoidales, $A_{4}$, como: $\frac{\mathbf{h}^{\frac{3}{2}}}{\mathbf{N}^{3}} \frac{\mathbf{1}}{\mathbf{1 2 5}}(1+9+25+49+81)$; así, una simple generalización nos permite escribir:

$$
A_{N}=\frac{h^{\frac{3}{2}}}{N^{3}} \sum_{k=1}^{N}(2 k-1)^{2}
$$

La expresión para la suma de los cuadrados de lo números impares es conocidd ${ }^{2}$ y nuestra aproximación trapezoidal queda: $A_{N}=\frac{h^{\frac{3}{2}}}{N^{3}} \frac{1}{3}(2 N+1) N(2 N+1)$ Finalmente el cálculo de Thabit se obtiene para $N \gg 1$, de este modo $A_{N} \rightarrow A$ el área del segmento de parábola, resultando:

$$
A=\frac{4}{3} h^{\frac{3}{2}} .
$$

Alternativamente, la suma de los cuadrados de los $\mathrm{N}$ primeros números naturales impares puede obtenerse de manera elemental, construyendo un "pirámide" (ver Fig. 4, que corresponde al caso $N=4$ ), que consta de "capas con cubos unitarios" dependientes de los cuadrados de los números enteros impares. Cada capa tiene espesor 1 (la unidad). El cálculo simple que sigue, es análogo al se realiza rutinariamente para calcular el número de estados cuánticos para una partícula en una caja $[11,12]$.

En el límite $N \gg 1$, la pirámide tiene una base $(2 N-1)^{2}$ y altura $N, \mathrm{y}$ su volumen, es decir la suma

\footnotetext{
1 En inglés, se usa la palabra "exhaustion". También se puede traducir como "agotamiento",

2 Una amigable aproximación a esta serie de sumas de enteros puede encontrarse en Ref. 10
} 


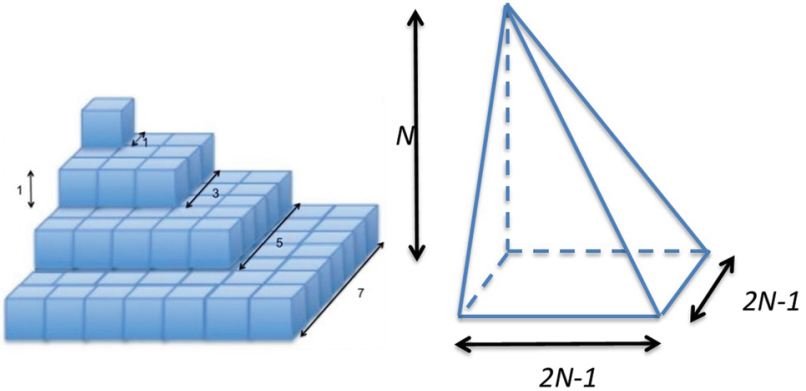

Figura 4: A la izquierda, la "pirámide" compuesta de cubos unitarios. A la derecha la pirámide para $N>>1$.

de los impares al cuadrado, es: $\frac{1}{3} N(2 N-1)^{2}$, que en nuestra aproximación, puede estimarse como $\frac{4}{3} N^{3}$. Este resultado coincide con lo deducido anteriormente (Ec. 2).

Si reconocemos que $h \sqrt{h}$ es el área del triángulo inscrito, se obtiene la propiedad que ya Arquímedes encontró: ... "For it is here shown that every segment bounded by a straight line an a right-angled cone (a parabola) is four-thirds of the triangle which has the same base and equal height with the segment, ... 3

\section{Comentarios finales}

Quizás la referencia a la cinemática pueda aparecer redundante o innecesaria para el tema de esta nota. Aunque eso es estrictamente correcto, el énfasis de esta aproximación está en la necesidad didáctica de unificar (o por lo menos, relacionar) conceptos usados en física y matemáticas. No dejaría de ser extraño que la parábola de la geometría analítica sea diferente a la de la caída libre.

Claramente el tratamiento de Thabit es un precursor de los procedimientos del cálculo infinitesimal [13]. Como es fácil notar, el inteligente método de Thabit puede ser reconstruido en notación contemporánea como el cálculo de la integral $\int \sqrt{x} d x$.

Aparentemente es la primera mención histórica al cálculo de una potencia fraccional de $x$. De facto, el área que estamos calculando es $2 h \sqrt{h}-\int_{-\sqrt{h}}^{\sqrt{h}} x^{2} d x=\frac{4}{3} h \sqrt{h}$, que coincide con la Ec. 2. Es recalcable la simplicidad que nos proporcional el cálculo infinitesimal.

Concluyendo, hemos recreado la demostración de Thabit de la cuadratura de la parábola, utilizando herramientas que nos proporciona el desarrollo habitual del estudio de proyectiles en cinemática. Este método tiene la ventaja didáctica de relacionar explícitamente conceptos de física y matemáticas.

Es también del caso resaltar la notable agudeza del pensamiento geométrico en los países de Oriente Medio, siglos antes que la herencia griega floreciera en Europa Occidental. El conocimiento de la obra de los pensadores árabes, ensancha nuestra visión histórica que culmina con

\footnotetext{
3 "Se ha demostrado todo segmento limitado por una línea recta en un cono recto (una parábola) es cuatro tercios del triángulo que tiene la misma base e igual altura que el segmento".
}

el surgimiento del renacimiento científico en el mundo occidental.

\section{Referencias}

[1] A. Sayili, ISIS 5135 (1960).

[2] S. Brentjes Historia Mathematica 16, 373 (1989).

[3] Archimedes The Quadrature of the Parabola,traduzido por T.L. Heath (Cambridge University Press Cambridge 1897).

[4] R. Rashed, in: Different Aspects of Islamic Culture (Unesco Publishing, Paris, 2001), vol. 4, Part. I

[5] G. Galileo Two New Sciences, traduzido por H Crew and A de Salvio (Dover, New York 1954) Hay una versión más reciente: Galileo G Two New Sciences Including Centers of Gravity and Force of Percussion, traducido por S Drake (Wall \& Emerson, Toronto 1989).

[6] A. Leahy Mathematics Magazine 87, 174 (2014).

[7] J. Wallis The Arthmetic of Infinitesimals (Springer, New York 2004).

[8] M.M. Munstersbjorn in: The Growth of Mathematical Knowledge (Eds. Springer Science, Doldrech, 2000).

[9] C. H. Wörner Rev. Bras. Ens. Fisica 34, 2305 (2012).

[10] https://culturacientifica.com/2016/11/16/masmatematicas-ver-tocar/.

[11] C. H. Wörner and E. Muñoz, Eur. J. Phys. 33, 1465 (2012).

[12] R.A. Serway, C.J. Moses and C.A. Moyer, Modern Physics (Thomson-Brooks/Cole, Belmont 2005), 3a. ed.

[13] A. Stoll, Repères IREM 11, 47 (1993), disponível em http://www.univ-irem.fr/exemple/reperes/ articles/11_article_71.pdf 